\title{
Effect of Anti-glaucoma Agents on Myopic Retinoschisis
}

\author{
Kyuhwan Jang, Junghwa Lee, Kyungmin Lee, Mi Jeung Kim, Joonhong Sohn \\ Department of Ophthalmology, Hangil Eye Hospital, Incheon, Korea
}

Purpose: To evaluate the effect of intraocular pressure (IOP)-lowering medications on myopic retinoschisis.

Methods: The medical records of 33 patients (36 eyes) with myopic retinoschisis associated with pathologic myopia were reviewed retrospectively. The patients were divided into two groups: the study group comprising patients undergoing treatment with anti-glaucoma medications for suspected glaucoma; the control group comprising patients who did not use any IOP lowering medications. The changes in retinoschisis in the two groups were compared using the Spectralis domain optical coherence tomography thickness map protocol.

Results: The study group included 18 eyes (17 patients), and the control group included 18 eyes (16 patients). There were no significant differences between the 6-month and 12-month improvement or aggravation rates of the two groups ( $p=0.513$ and 0.137 , respectively). However, after 18 months, the aggravation rate of retinoschisis was significantly lower in the study group $(p=0.003)$. The improvement / aggravation rate was $58.33 \% / 16.67 \%$ in the study group and $0 \% / 57.14 \%$ in the control group.

Conclusions: The use of IOP-lowering medications for more than a year may be useful for the management of retinoschisis associated with pathologic myopia.

Key Words: Glaucoma, Intraocular pressure, Myopia, Retinoschisis

Myopic retinoschisis is one of the main causes of visual loss in patients with high myopia, and the prevalence of myopic retinoschisis is estimated to be $9 \%$ to $20 \%$ in eyes with pathological myopia with posterior staphyloma [1-3].

According to previous studies, myopic retinoschisis can progress to severe complications, such as full-thickness macular holes and foveal detachment [4,5]. Additionally, surgical interventions are the only currently available treatment options for myopic retinoschisis; however, the

Received: July 6, 2020 Final revision: September 10, 2020 Accepted: September 24, 2020

Corresponding Author: Joonhong Sohn, PhD. Department of Ophthalmology, Hangil Eye Hospital, 35 Bupyeong-daero, Bupyeong-gu, Incheon 21388, Korea. Tel: 82-32-503-3322, Fax: 82-32-504-3322, E-mail: jhsohn19@hanafos.com results of surgical treatments have not been completely satisfactory. Further, we can only observe aggravation without any treatment until surgery [6-9].

Previous studies have shown that axial length decreases when trabeculectomy is performed in patients with high myopia [10-13], and animal studies have also reported that axial length decreases when intraocular pressure (IOP) is lowered [14-16]. Another study revealed that IOP and increased axial length affected the progression of myopic retinoschisis [17]. Based on these findings, we believe that IOP may play an important role in the progression of retinoschisis.

Therefore, the purpose of this study was to evaluate the effects of IOP-lowering eye drops on the progression of myopic retinoschisis. 


\section{Materials and Methods}

We retrospectively reviewed the medical records of patients with high myopia and myopic retinoschisis treated at Hangil Eye Hospital between April 2014 and May 2018. This study adhered to the tenets of the Declaration of Helsinki, and it was approved by the institutional review board of Hangil Eye Hospital (IRB-19001). The requirement to obtain informed consent from study participants was waived by the institutional review board given the retrospective nature of the study.

The inclusion criteria were as follows: (1) patients who had retinoschisis without any comorbidities, such as a macular hole or retinal detachment (RD) diagnosed using spectral-domain optical coherence tomography (SD-OCT), and (2) patients with high myopia, that is, a spherical equivalent of $>-6$ diopters (D) or an axial length of 26.0 $\mathrm{mm}$. The exclusion criteria were as follows: (1) patients with a history of vitrectomy, (2) patients who underwent cataract or glaucoma surgery within 6 months before enrollment in the study, and (3) patients who were already using IOP-lowering medications, including glaucoma patients.

We divided the included patients into two groups: (1) the study group, which comprised patients who were treated with IOP-lowering medications for suspected glaucoma during follow-up, and (2) the control group, which comprised patients who were not treated with any eye drops.

All patients underwent ophthalmologic examinations,
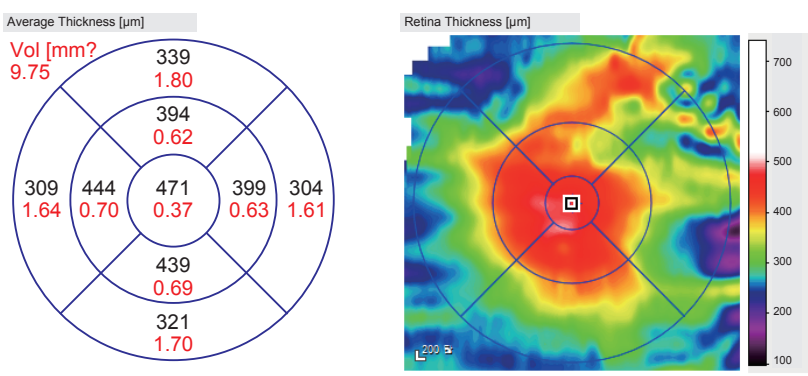

Fig. 1. Spectral-domain optical coherence tomography thickness mapping protocol. Location and thickness of the retina on spectral-domain optical coherence tomography. The thicknesses at the center and 1,3, and $6 \mathrm{~mm}$ away from the center are indicated. The total retinal thickness was defined as the sum of the thicknesses of all the sections, and the thickness of each sector (nasal, temporal, superior, and inferior), except the center, was calculated as the thickness between 1 and $3 \mathrm{~mm}$ and the thickness between 3 and $6 \mathrm{~mm}$. including best-corrected visual acuity (BCVA), manual and automatic refractive examinations (ARK-530A, Nidek, Gamagori, Japan), fundus examinations (Optos PLC; Dunfermline, Scotland, UK), tonometry (NT-530P; Nidek, Aichi, Japan), and SD-OCT ver. 5.3.2.0 (Heidelberg Engineering, Heidelberg, Germany).

The progression of myopic retinoschisis was retrospectively analyzed using OCT at the initial visit and 6, 12, and 18 months. The progression of retinoschisis was evaluated using the following method.

An SD-OCT thickness mapping protocol was used to measure retinal thickness 1,3 , and $6 \mathrm{~mm}$ from the central fovea. Using this protocol, the macula was separated into the following five sections: (1) central, (2) lateral, (3) nasal, and (4) upper and (5) lower sections (Fig. 1). Horizontal lines that penetrated the center of the macula were used to measure the central retinal thickness, and the thickness was measured between the innermost bright line, which corresponded to the inner limiting membrane, and the outermost line, which corresponded to the retinal pigment epithelium [18]. The automated segmentation error was corrected by two authors, and disagreement was adjusted by the senior author.

Considering the error of the measured value [19-21], we defined the improvement in myopic retinoschisis as a decrease in the total retinal thickness by more than $10 \%$ in two or more out of five sections without an increase in volume of more than $10 \%$ in any other sections. The opposite case was considered an aggravation.

Statistical analyses were performed using IBM SPSS Statistics ver. 23 (IBM Corp., Armonk, NY, USA). The Mann-Whitney and Pearson's chi-squared tests were used to compare the data of the two groups.

\section{Results}

We retrospectively analyzed 36 eyes of 33 patients with myopic retinoschisis during the study period. The study group comprised 18 eyes of 17 patients, and the control group comprised 18 eyes of 16 patients.

The mean age of patients in the study group at the initial visit was $68.44 \pm 8.60$ years; IOP was $16.89 \pm 3.45 \mathrm{mmHg}$, visual acuity (VA) was $0.50 \pm 0.21$ (Snellen), the spherical equivalent (SE) was $-4.40 \pm 4.77 \mathrm{D}$, the axial length was $29.42 \pm 1.38 \mathrm{~mm}$, and the follow-up duration was $411.42 \pm$ 219.86 days. There were no significant differences between 
Table 1. Baseline clinical characteristics of the study and control groups

\begin{tabular}{lccc}
\hline Parameter & Study group & Control group & $p$-value \\
\hline Number (male : female) & $18(1: 17)$ & $18(2: 16)$ & $0.564^{*}$ \\
Age (yr) & $68.44 \pm 8.60(53$ to 84$)$ & $63.6 \pm 8.31(53$ to 81$)$ & $0.104^{\dagger}$ \\
Initial IOP (mmHg) & $16.89 \pm 3.45(10$ to 21$)$ & $16 \pm 3.58(8$ to 21$)$ & $0.501^{\dagger}$ \\
Visual acuity (Snellen) & $0.50 \pm 0.21(0.15$ to 0.8$)$ & $0.58 \pm 0.29(0.03$ to 1.0$)$ & $0.406^{\dagger}$ \\
Spherical equivalent (D) & $-4.40 \pm 4.77(-16.50$ to -0.40$)$ & $-5.12 \pm-5.5(-17$ to -1$)$ & $0.743^{\dagger}$ \\
Axial length (mm) & $29.42 \pm 1.38(27.85$ to 32.00$)$ & $29.79 \pm 1.10(28.31$ to 2.32$)$ & $0.334^{\dagger}$ \\
\hline
\end{tabular}

Values are presented as mean \pm standard deviation (range).

$\mathrm{IOP}=$ Intraocular pressure; $\mathrm{D}=$ diopters.

"Pearson's chi-square test; ${ }^{\dagger}$ Mann-Whitney test.

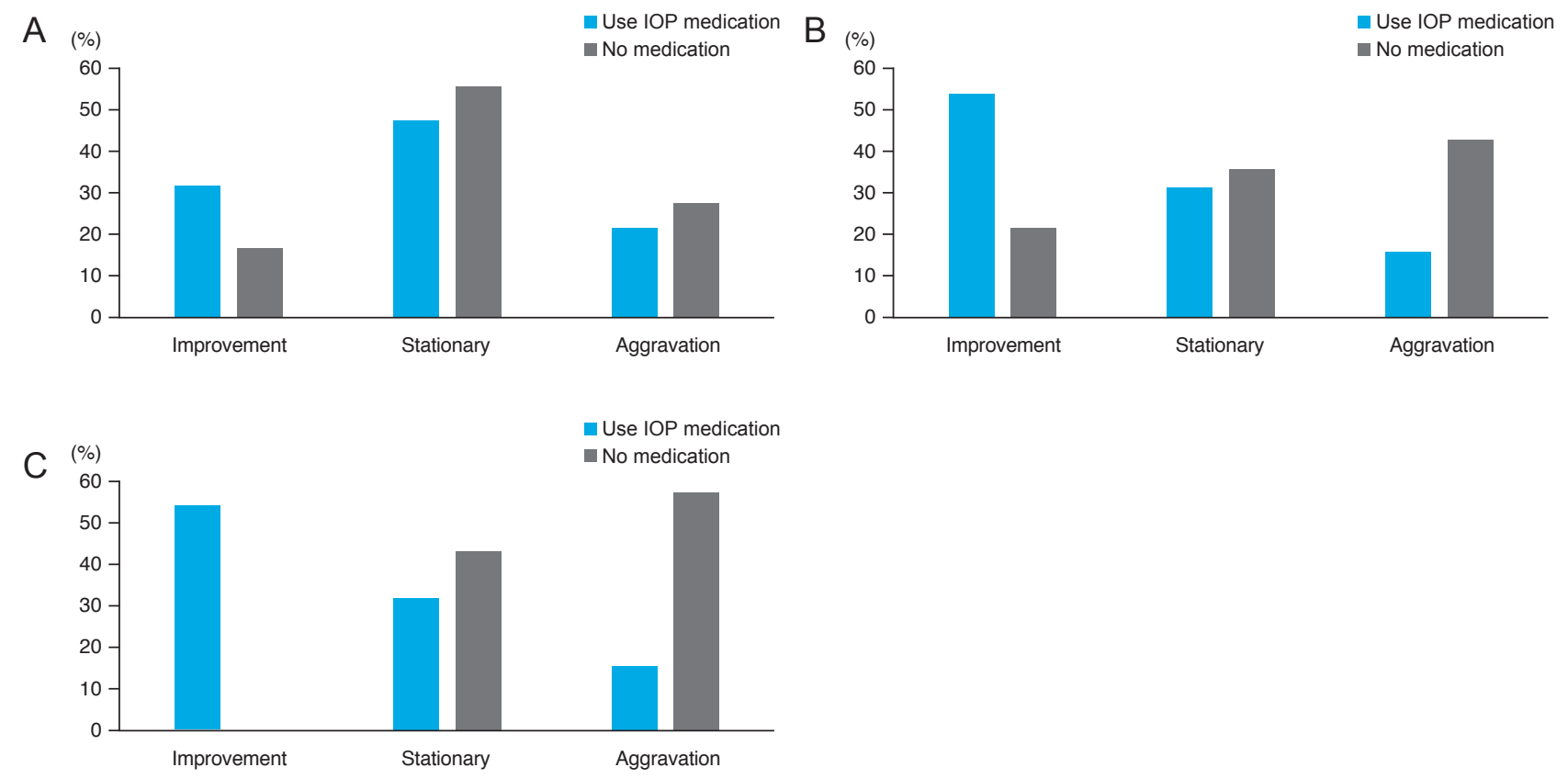

Fig. 2. Comparison of the progression rates of the study and control groups. (A) Comparison of progression rates of the group that used intraocular pressure (IOP) medication and the group that did not use any drug after 6 months. There was no significant difference between the degrees of improvement of the two groups ( $p=0.513$, Pearson's chi-squared test). (B) Comparison of the progressions of the group that used IOP medication and the group that did not use any drugs after 12 months. There was no significant difference between the degrees of improvement of the two groups ( $p=0.137$, Pearson's chi-squared test). (C) Comparison of progressions of the group that used IOP medication and the group that did not use any drug after 18 months. There was a significant difference in the degree of improvement between the two groups ( $p=0.03$, Pearson's chi-squared test).

the ages, IOPs, visual acuities, SEs, and axial lengths of the study and the control groups at the initial visit (Table 1). There was also no difference between the follow-up durations of the two groups (Table 1).

At the 6 months, there were no significant differences between the number of patients with improvement in retinoschisis, stationary retinoschisis, and aggravation of reti- noschisis in the two groups ( $p=0.513$, Pearson's chisquared test) (Fig. 2A). After 1 year, 58.33\% of patients showed improvement in retinoschisis in the study group, and the aggravation rate was $16.67 \%$. On the contrary, the improvement and aggravation rates of the control group were $21.43 \%$ and $42.86 \%$, respectively ( $p=0.137$, Pearson's chi-square test) (Fig. 2B). After the 18 months, there was a 
Table 2. Comparison of parameters before and after the use of intraocular pressure-lowering medications in the study group

\begin{tabular}{lccc}
\hline Parameter & $\begin{array}{c}\text { Values before } \\
\text { medication } \\
\text { (range) }\end{array}$ & $\begin{array}{c}\text { Values at } \\
\text { follow-up after } \\
18 \text { months } \\
\text { (range) }\end{array}$ & $p$-value* \\
\hline $\begin{array}{c}\text { Visual acuity } \\
\text { (Snellen) }\end{array}$ & $0.50 \pm 0.21$ & $0.55 \pm 0.25$ & 0.572 \\
$\begin{array}{c}\text { Spherical equivalent } \\
\text { (diopter) }\end{array}$ & $-4.40 \pm 4.77$ & $-3.65 \pm 4.35$ & 0.692 \\
$\begin{array}{c}\text { Intraocular pressure } \\
\text { (mmHg) }\end{array}$ & $16.89 \pm 3.45$ & $13.58 \pm 3.42$ & 0.017 \\
\hline
\end{tabular}

Values are presented as mean \pm standard deviation.

*Mann-Whitney test.

Table 3. Comparison of the study and control groups at 18 months follow-up

\begin{tabular}{lccc}
\hline Parameter & $\begin{array}{c}\text { Study group } \\
\text { value (range) }\end{array}$ & $\begin{array}{c}\text { Control group } \\
\text { value (range) }\end{array}$ & $p^{\text {-value }}$ \\
\hline $\begin{array}{l}\text { Visual acuity } \\
\text { (Snellen) }\end{array}$ & $0.55 \pm 0.25$ & $0.74 \pm 0.27$ & 0.085 \\
$\begin{array}{l}\text { Spherical equivalent } \\
\text { (diopter) }\end{array}$ & $-3.65 \pm 4.35$ & $-4.96 \pm 5.13$ & 0.527 \\
$\begin{array}{l}\text { Intraocular pressure } \\
(\mathrm{mmHg})\end{array}$ & $13.58 \pm 3.42$ & $15.14 \pm 2.48$ & 0.085 \\
\hline
\end{tabular}

Values are presented as mean \pm standard deviation.

*Mann-Whitney test.

significant difference between the progressions of retinoschisis. The improvement and aggravation rates of patients in the study group were $58.33 \%$ and $16.67 \%$, respectively; however, those of the patients in the control group were $0 \%$ and $57.14 \%$, respectively ( $p=0.003$, Pearson's chi-squared test) (Fig. 2C, 3A-3D, 4A-4D). In the study group, only one patient developed macular hole (MH) and underwent vitrectomy after 18 months of follow-up. In the control group, no one developed a macular hole or $\mathrm{RD}$, and there was no significant difference between the two groups ( $p=1.00$, Fisher's exact test). The 18-month changes in VA $(p=0.274)$, IOP $(p=0.145)$, and SE $(p=0.705)$ showed significant differences.

There was a significant difference between the IOP at the initial visit and the IOP at the last follow-up in the study group (Table 2); however, there were no significant differences between the VAs and SEs at the initial visit and the last follow-up in the study group (Table 3). The IOP-lowering medications used in this study were fixed
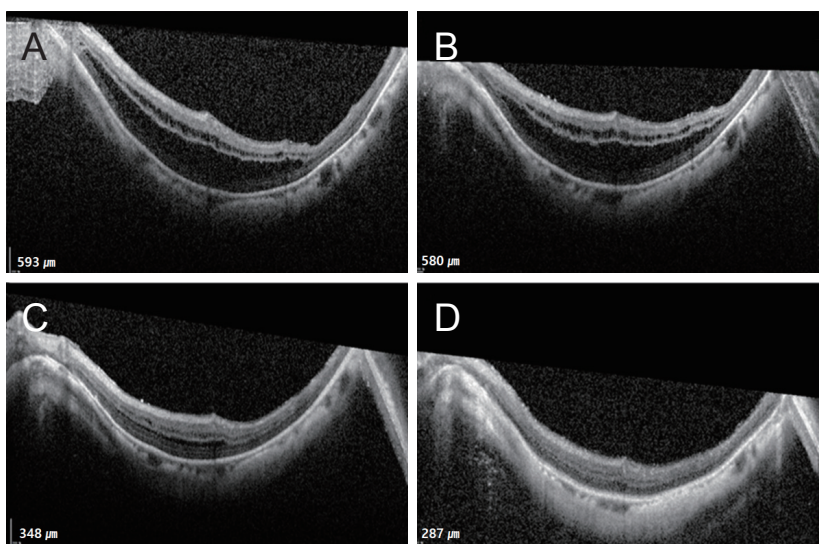

Fig. 3. A 70s female with myopic retinoschisis. Initial best-corrected visual acuity was Snellen 0.4, intraocular pressure (IOP) was $19 \mathrm{mmHg}$, and spherical equivalent was -1.75 diopters due to cataract surgery. The thickness of the retinoschisis region was 593 $\mu \mathrm{m}$. (A) Optical coherence tomography findings before the use of eye drops. Retinoschisis and posterior staphyloma were observed. (B) After 6 months of IOP medication use, there was no significant difference. (C) After 12 months of IOP medication use, the retinoschisis improved. The thickness of the retinoschisis region decreased to $348 \mu \mathrm{m}$. (D) After 18 months of IOP medication use, retinoschisis improved considerably. The thickness of the schisis region significantly decreased to $287 \mu \mathrm{m}$. IOP decreased to 16 $\mathrm{mmHg}$, and best-corrected visual acuity and spherical equivalent did not change.
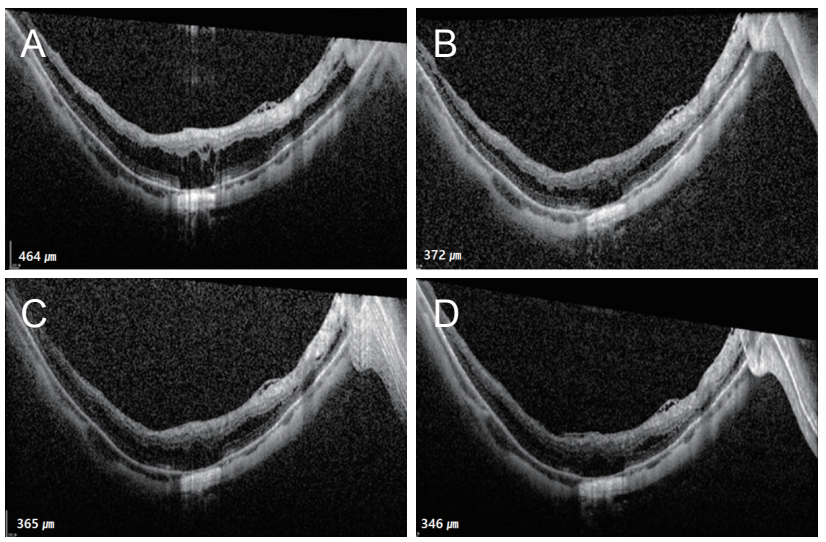

Fig. 4. A 50s female with myopic retinoschisis. Initial best-corrected visual acuity was Snellen 0.8 , intraocular pressure (IOP) was 21 , and spherical equivalent was -1.00 diopters due to cataract surgery. The thickness of the retinoschisis area was $464 \mu \mathrm{m}$. (A) Optical coherence tomography findings before the use of eye drops. Retinoschisis and posterior staphyloma were observed. (B) After 6 months of IOP medication use, the retinoschisis improved. The thickness of retinoschisis decreased to $372 \mu \mathrm{m}$. (C) After 12 months of IOP medication use, there was no difference. (D) After 18 months of IOP medication use, retinoschisis improved considerably. The thickness decreased to $346 \mu \mathrm{m}$. IOP decreased to $12 \mathrm{mmHg}$, best-corrected visual acuity increased to Snellen 1.0, and emmetropia was attained. 
combination drugs (CA-inhibitor $+\beta$-blocker; 10 eyes), $\beta$-blockers (six eyes), prostaglandin analogs (one eye), and CA-inhibitors (one eye). There were no significant differences between the VA, IOP, and SE changes stratified by medication ( $p=0.414,0.091$, and 0.829 , Kruskal Wallis).

\section{Discussion}

Several previous studies have revealed that myopic retinoschisis is progressive. Specifically, Shimada et al. [22] reported the natural history of myopic retinoschisis in patients without an initial macular hole or RD. During the follow-up, $25 \%$ of the eyes developed an $\mathrm{MH}$, and $25 \%$ developed $\mathrm{RD}$ without a macular hole. The remaining 50\% did not develop any other complications; however, the thickness of the macula significantly increased. Another study demonstrated that retinoschisis progressed in 20 out of 29 eyes over 31.2 months, and the other 9 eyes remained stable. Vitrectomy was performed in 11 of the 20 worsened eyes, but 3 eyes developed a macular hole after surgery [4]

Additionally, Shimada et al. [5] reported that eight out of 207 eyes $(3.9 \%)$ showed a decrease or complete resolution of macular retinoschisis within $36.2 \pm 6.2$ months, and 24 (11.6\%) eyes experienced the aggravation of myopic traction maculopathy. However, the study conducted by Shimada et al. [5] also included patients with a mild type of retinoschisis, and the rate of aggravation was significantly higher in eyes with more extensive macular retinoschisis than in eyes with mild retinoschisis $(42.9 \%)$. Similar to the results of that study, our study demonstrated that retinoschisis progressed in $57.14 \%$ of patients who did not use IOP-lowering medications and $16.67 \%$ of patients who were administered IOP-lowering medications after 18 months.

Currently, surgical interventions are the only available treatments for patients with retinoschisis. However, complications, such as $\mathrm{MH}$, may occur after surgery, and the burden of the surgery may be problematic [6-9]. In addition, the patients without any treatment before surgery were observed. However, in the current study, IOP-lowering medications were administered to patients with myopic retinoschisis, and our results demonstrated that there were significant differences between the progressions of retinoschisis in the study and control groups. Although there were no differences in the status of retinoschisis in the two groups after 6 months, we observed significant differences in the retinoschisis status in the two groups after 1.5 years. After 18 months, $58.33 \%$ showed an improvement, $25.00 \%$ showed no change, and $16.67 \%$ showed aggravation. Overall, the improvement rate was higher and the aggravation rate was lower in this study than in previous studies, and these findings suggest that lowering IOP may significantly slow down the natural course of or improve myopic retinoschisis.

There is no established explanation for the improvement in retinoschisis associated with IOP-lowering medication, but the findings of previous studies on the effects of IOP medication on myopia aggravation may be foundational.

In 2000, Jin and Stjernschantz [15] injected 100 ng of latanoprost into the vitreous cavity of chickens twice a week and found that the elongation of the eye was more attenuated in chickens that received latanoprost than in chickens that received isotonic saline. Additionally, Liu et al. [16] reported that treatment with $0.1 \%$ brimonidine alone, $0.2 \%$ brimonidine alone, or brimonidine combined with $2 \%$ pirenzepine effectively inhibited the progression of myopia by improving the refractive error and shortening the axial length. They suggested that the IOP changes may inform potential treatment strategies for progressing myopia. Finally, El-Nimri and Wildsoet [14] reported that myopia aggravation in guinea pigs that were administered with latanoprost was lower than that in the control group; therefore, myopia aggravation may be lowered with significant IOP reduction.

Several studies have shown a relationship among IOP, axial length, and myopia aggravation. Several studies reported that IOP was significantly higher in the moderate myopia group than in the emmetropia group [17,23,24]. Additionally, axial length was lower after trabeculectomy than preoperatively $[10,12,13]$. IOP provides the force for stretching the scleral walls, and these forces play an important role in maintaining the sclera and the normal eye status. However, in myopic eyes, IOP may also promote axial length elongation, which may lead to myopic aggravation. As myopia progresses, the sclera becomes weaker and thinner, and it becomes more susceptible to changes caused by IOP $[25,26]$. A few studies have also suggested that age-related elongation of eyes and the expansion of staphylomas may underlie the development of myopic retinoschisis; therefore, intraocular and extraocular wall factors play important roles $[14,27]$. These findings demon- 
strate that IOP is directly related to the aggravation of myopia, myopic retinoschisis, and an increase in axial length.

In this study, we found that lowering IOP with medications in patients with pathological myopia may inhibit the increase in axial length and control the aggravation of myopic retinoschisis. Although we were unable to compare changes in axial length before and after the use of IOP-lowering medications, there were no significant changes in SE for more than one year; therefore, this treatment may have inhibited axial length elongation during the study period. Although there was no significant difference in the status of retinoschisis in the study and control groups during the first six months of treatment, there was a significant difference in the aggravation of retinoschisis in the two groups from the first year of treatment. This suggests that IOP-lowering medications should be used for at least 1 year to achieve optimum therapeutic effects in patients with myopic retinoschisis.

This study had limitations. The sample was relatively small (33 patients and 36 eyes), and we used various types of IOP-lowering medications. However, there were no differences between the VAs, SEs, or IOPs of the patients who received different medications. Another limitation was that the underlying diseases in the two groups were not identical. The study group consisted of myopic retinoschisis patients with suspected glaucoma, but the control group had no sign of glaucoma. In addition, the axial length was not measured in the two groups; thus, the change in axial length was inferred from the change in SE. Although this study has limitations due to its retrospective design, we believe that it is worth reporting. However, a prospective and randomized study is needed.

This is the first comparative control study to observe the effects of IOP-lowering medications on pathologic myopic retinoschisis in patients with glaucoma. Overall, our findings suggest that IOP-lowering medications may be beneficial in the management of retinoschisis associated with pathological myopia.

\section{Conflict of Interest}

No potential conflict of interest relevant to this article was reported.

\section{References}

1. Baba T, Ohno-Matsui K, Futagami S, et al. Prevalence and characteristics of foveal retinal detachment without macular hole in high myopia. Am J Ophthalmol 2003;135:338-42.

2. Panozzo G, Mercanti A. Optical coherence tomography findings in myopic traction maculopathy. Arch Ophthalmol 2004;122:1455-60.

3. Takano M, Kishi S. Foveal retinoschisis and retinal detachment in severely myopic eyes with posterior staphyloma. Am J Ophthalmol 1999;128:472-6.

4. Gaucher D, Haouchine B, Tadayoni R, et al. Long-term follow-up of high myopic foveoschisis: natural course and surgical outcome. Am J Ophthalmol 2007;143:455-62.

5. Shimada N, Tanaka Y, Tokoro T, Ohno-Matsui K. Natural course of myopic traction maculopathy and factors associated with progression or resolution. Am J Ophthalmol 2013;156:948-57.

6. Ikuno Y, Sayanagi K, Ohji M, et al. Vitrectomy and internal limiting membrane peeling for myopic foveoschisis. Am J Ophthalmol 2004;137:719-24.

7. Kanda S, Uemura A, Sakamoto Y, Kita H. Vitrectomy with internal limiting membrane peeling for macular retinoschisis and retinal detachment without macular hole in highly myopic eyes. Am J Ophthalmol 2003;136:177-80.

8. Rey A, Jurgens I, Maseras X, Carbajal M. Natural course and surgical management of high myopic foveoschisis. Ophthalmologica 2014;231:45-50.

9. Rizzo S, Giansanti F, Finocchio L, et al. Vitrectomy with internal limiting membrane peeling and air tamponade for myopic foveoschisis. Retina 2019;39:2125-31.

10. Kara N, Baz O, Altan C, et al. Changes in choroidal thickness, axial length, and ocular perfusion pressure accompanying successful glaucoma filtration surgery. Eye (Lond) 2013;27:940-5.

11. Popa-Cherecheanu A, Iancu RC, Schmetterer L, et al. Intraocular pressure, axial length, and refractive changes after phacoemulsification and trabeculectomy for open-angle glaucoma. J Ophthalmol 2017;2017:1203269.

12. Saeedi O, Pillar A, Jefferys J, et al. Change in choroidal thickness and axial length with change in intraocular pressure after trabeculectomy. Br J Ophthalmol 2014;98:976-9.

13. Usui S, Ikuno Y, Uematsu S, et al. Changes in axial length and choroidal thickness after intraocular pressure reduction resulting from trabeculectomy. Clin Ophthalmol 2013;7:1155-61. 
14. El-Nimri NW, Wildsoet CF. Effects of topical latanoprost on intraocular pressure and myopia progression in young guinea pigs. Invest Ophthalmol Vis Sci 2018;59:2644-51.

15. Jin N, Stjernschantz J. Effects of prostaglandins on form deprivation myopia in the chick. Acta Ophthalmol Scand 2000;78:495-500.

16. Liu Y, Wang Y, Lv H, et al. $\alpha$-adrenergic agonist brimonidine control of experimentally induced myopia in guinea pigs: a pilot study. Mol Vis 2017;23:785-98.

17. Nomura H, Ando F, Niino N, et al. The relationship between age and intraocular pressure in a Japanese population: the influence of central corneal thickness. Curr Eye Res 2002;24:81-5.

18. Cheng C, Teo K, Tan CS, et al. Myopic retinoschisis in Asians: structural features and determinants of visual acuity and prognostic factors for progression. Retina 2016;36:717-26.

19. Chung H, Lee K, Hwang DJ, et al. Prediction of morphologic deterioration in patients with lamellar macular holes. Retina 2016;36:1699-706.

20. Polito A, Del Borrello M, Isola M, et al. Repeatability and reproducibility of fast macular thickness mapping with stratus optical coherence tomography. Arch Ophthalmol 2005;123:1330-7.
21. Theodossiadis PG, Grigoropoulos VG, Emfietzoglou I, et al. Evolution of lamellar macular hole studied by optical coherence tomography. Graefes Arch Clin Exp Ophthalmol 2009;247:13-20.

22. Shimada N, Ohno-Matsui K, Baba T, et al. Natural course of macular retinoschisis in highly myopic eyes without macular hole or retinal detachment. Am J Ophthalmol 2006;142:497-500.

23. Oner V, Tas M, Ozkaya E, Oruc Y. Effect of pathological myopia on biomechanical properties: a study by ocular response analyzer. Int J Ophthalmol 2015;8:365-8.

24. Shen M, Fan F, Xue A, et al. Biomechanical properties of the cornea in high myopia. Vision Res 2008;48:2167-71.

25. Cahane M, Bartov E. Axial length and scleral thickness effect on susceptibility to glaucomatous damage: a theoretical model implementing Laplace's law. Ophthalmic Res 1992;24:280-4.

26. Nickla DL. Ocular diurnal rhythms and eye growth regulation: where we are 50 years after Lauber. Exp Eye Res 2013;114:25-34.

27. Wu PC, Chen YJ, Chen YH, et al. Factors associated with foveoschisis and foveal detachment without macular hole in high myopia. Eye (Lond) 2009;23:356-61. 